\title{
Neuroprotective effects of Gua Lou Gui Zhi decoction against glutamate-induced apoptosis in BV-2 cells
}

\author{
ZUANFANG LI ${ }^{1}$, HAIXIA HU ${ }^{1}$, RUHUI LIN ${ }^{1}$, JINGJIE MAO $^{1}$, XIAOQIN ZHU ${ }^{1}$, \\ ZHENFENG HONG ${ }^{1}$, JING TAO ${ }^{2}$, YUN ZHANG ${ }^{2,3}$ and LIDIAN CHEN ${ }^{2}$ \\ ${ }^{1}$ Fujian Academy of Integrative Medicine, ${ }^{2}$ College of Rehabilitation Medicine, Fujian University of \\ Traditional Chinese Medicine, Fuzhou, Fujian 350108; ${ }^{3}$ Graduate School of Heilongjiang University of Chinese Medicine, \\ Heilongjiang University of Chinese Medicine, Harbin, Heilongjiang 150040, P.R. China
}

Received September 11, 2013; Accepted December 19, 2013

DOI: $10.3892 /$ ijmm.2013.1612

\begin{abstract}
Gua Lou Gui Zhi decoction (GLGZD), a traditional Chinese medicine consisting of different herbal medicines, has been used for centuries in the treatment of muscular spasticity following stroke, epilepsy or spinal cord injury. However, the precise mechanisms involved remain poorly understood. In the present study, we investigated the neuroprotective effects of GLGZD on glutamate-induced apoptosis in cultured BV-2 cells, as well as the underlying mechanisms. A 3-(4,5-dimethylthiazol2-yl)-2,5-diphenyltetrazolium bromide (MTT) assay was applied to assess the viability of the cells. An Annexin V/propidium iodide (PI) assay was utilized to analyze cellular apoptosis. Mitochondrial membrane potential (MMP) was evaluated by flow cytometry and laser scanning confocal microscopy. The gene and protein expression of the apoptosis-related genes, Bcl-2 and Bax, was analyzed by RT-PCR and western blot analysis, respectively. Furthermore, the expression of cleaved caspase-3 protein was detected by immunofluorescence. Glutamate treatment induced the loss of BV-2 cell viability, which was associated with an increase in the apoptotic rate, as well as an increase in the $\mathrm{Bax} / \mathrm{Bcl}-2$ ratio and the extracellular levels of cleaved caspase- 3 . Treatment with GLGZD significantly reversed these phenotypes, with its maximum protective effects observed at the concentration of $1,000 \mu \mathrm{g} / \mathrm{ml}$. These results indicate that GLGZD protects $\mathrm{BV}-2$ cells from glutamate-induced cytotoxicity. These protective effects may be ascribed to its anti-apoptotic activities, in part, associated with the decrease in the $\mathrm{Bax} / \mathrm{Bcl}-2$ ratio and caspase- 3 expression, as well as with the stability of high mitochondrial membrane potential.
\end{abstract}

Correspondence to: Professor Lidian Chen, College of Rehabilitation Medicine, Fujian University of Traditional Chinese Medicine, 1 Huatuo Road, Minhou Shangjie, Fuzhou, Fujian 350108, P.R. China

E-mail: cld@fjtcm.edu.cn

Key words: Gua Lou Gui Zhi decoction, BV-2 cells, glutamate, apoptosis

\section{Introduction}

Microglia, the resident innate immune cells of the brain, have been suggested to play a role in host defense and tissue repair in the central nervous system (CNS) and CNS-associated diseases, such as Parkinson's, Alzheimer's and Huntington's diseases (1). BV-2 cells are a common microglial cell line that has been widely used to study inflammatory and necrotic reactions during the course of neurological diseases (2-4).

Traditional Chinese Medicine (TCM), which has a history of more than three thousand years, is based on treatments using compounds extracted from the natural environment (plants). There are many herbal prescriptions for the treatment of CNS-associated diseases. Gua Lou Gui Zhi decoction (GLGZD), consists of extracts of Trichosanthis Radix, Ramulus Cinnamomi, Paeonia lactiflora, Glycyrrhiza, Zingiber officinale Roscoe and Fructus Jujubae (5-7). This treatment has been formulated from the time of the Eastern Han Dynasty (25-220 AD), and has typically been used in the treatment of muscular spasticity following stroke, epilepsy, or spinal cord injury (5-7). However, the precise mechanisms responsible for its neuroprotective and anti-spasticity effects remain poorly understood.

Neuronal apoptosis, a form of programmed cell death that may serve in the regulation of nervous system development, is an important mechanism of neuronal death in many models of acute and chronic neurological disorders $(8,9)$. Glutamate, a major excitatory amino acid neurotransmitter in the CNS, mediates several physiological processes by engaging the ionotropic glutamate receptor and the metabotropic glutamate receptor (10). However, dysfunction of these glutamate transporters may be a major contributing factor to the increase in extracellular glutamate concentration and resulting excitotoxicity. Furthermore, the excess stimulation of glutamate receptors can induce neuroinflammation and eventual neurodegeneration (11). Therefore, glutamate toxicity has been implicated in several acute and chronic neurological disorders, such as cerebral ischemia, stroke, epilepsy, Alzheimer's disease and Parkinson's disease (1,10-13).

Thus, in this study, we used glutamate to induce damage to BV-2 cells, and investigated the protective effects of GLGZD on this cell model, as well as the underlying mechanisms involved. 


\section{Materials and methods}

GLGZD water extract. The prescription of GLGZD was first recorded in 'Jin Gui Yao Lue', a medical book written by Zhongjing Zhang of the Eastern Han Dynasty during the first century (25-220 AD). The formula consists of six crude drugs, including Trichosanthis Radix, Ramulus Cinnamomi, Paeonia lactiflora, Glycyrrhiza, Zingiber officinale Roscoe and Fructus Jujubae at a ratio of 3:3:3:2:3:3. Dried crude drugs were purchased from Tongrentang Chinese Medicine Pharm (Fuzhou, China), a famous and time-honored pharmaceutical brand in the TCM industry in China. They were identified and confirmed by the College of Pharmacology, Fujian University of Traditional Chinese Medicine, Fuzhou, China. The formula was prepared by boiling the herbs in water. After the first decoction $(2 \mathrm{~h})$, the suspension was filtered and water was added for the second decoction $(1 \mathrm{~h})$. The filtered and mixed suspension from the two decoctions was concentrated under vacuum by using a rotary evaporator to a final concentration of $1.16 \mathrm{~g} / \mathrm{ml}$. The samples were then stored at $-20^{\circ} \mathrm{C}$ before use.

Cell culture and treatments. The cells were cultured in DMEM/high glucose medium supplemented with $10 \%$ fetal bovine serum (FBS), $100 \mathrm{U} / \mathrm{ml}$ penicillin and $100 \mu \mathrm{g} / \mathrm{ml}$ streptomycin solution at $37^{\circ} \mathrm{C}$ under an atmosphere of $5 \% \mathrm{CO}_{2}$ (Thermo Fisher Scientific, Waltham, MA, USA). One day prior to treatment, the culture medium was changed to DMEM/high glucose medium with $0.5 \%$ FBS in order to reduce the serum effect. Twenty-four hours after seeding, the medium was renewed with one of the three types of fresh culture medium (medium without glutamate, with $30 \mathrm{mM}$ glutamate, and $30 \mathrm{mM}$ glutamate plus various concentrations of GLGZD), and incubated for $24 \mathrm{~h}$. In a single experiment each treatment was performed in triplicate.

Cell viability. Cells were seeded into 96-well plates at a concentration of $5-6 \times 10^{4}$ cells $/ \mathrm{ml}$. Cell viability was evaluated with a 3-(4,5-dimethylthiazol-2-yl)-2,5-diphenyltetrazolium bromide (MTT) assay. The MTT assay was performed as follows: $20 \mu \mathrm{l}$ of $5 \mathrm{mg} / \mathrm{ml}$ MTT dissolved in phosphate-buffered saline (PBS) was added to each individual well followed by incubation at $37^{\circ} \mathrm{C}$ for $4 \mathrm{~h}$. The solution was then removed, and the produced formazan was solubilized in $100 \mu$ l dimethyl sulfoxide (DMSO). Absorbance was measured at $570 \mathrm{~nm}$ using an automated microplate reader (Bio-Rad Laboratories, Hercules, CA, USA). Cell viability was expressed as a percentage of the control culture value.

Analysis of cell morphology. Cells were seeded into 6-well microplates. Following treatment for $24 \mathrm{~h}$, the cells were fixed for $10 \mathrm{~min}$ with $4 \%$ paraformaldehyde in PBS, washed with PBS, then visualized and photographed under a phase contrast microscope (Leica, Wetzlar, Germany).

Analysis of apoptosis. The apoptosis of BV-2 cells was determined by flow cytometry on a FACSCalibur flow cytometer (BectonDickinson, Franklin Lakes, NJ, USA) with an Annexin V-FITC Apoptosis Detection kit (KeyGen Biotech, Nanjing, China). Staining was performed according to the manufacturer's instructions. The percentage of cells found in early apoptosis was calculated by counting the number of Annexin V-positive and propidium iodide (PI)-negative cells. The percentage of cells found in late apoptosis was calculated by counting the number of Annexin V-positive and PI-positive cells.

Analysis of mitochondrial membrane potential (MMP). The JC-1 assay kit (KeyGen Biotech) was employed to measure the MMP of BV-2 cells according to the manufacturer's instructions. Briefly, the cells were seeded into 6-well plates and exposed to glutamate or GLGZD for $24 \mathrm{~h}$. Thereafter, the cells were harvested and resuspended in a mixture of $500 \mu \mathrm{l}$ culture medium and $500 \mu \mathrm{JC}-1$ staining fluid, and incubated in the dark at $37^{\circ} \mathrm{C}$ for $20 \mathrm{~min}$. Following two washes with JC-1 staining buffer and incubation in DMEM, the cells were analyzed by flow cytometry. Mitochondria containing red JC-1 aggregates in healthy cells were detectable in the FL-2 channel, and those containing green JC-1 monomers in apoptotic cells were detectable in the FL-1 channel. The values of MMP staining from each sample were expressed as the ratio of red fluorescence intensity over green fluorescence intensity.

We also evaluated the MMP of BV-2 cells in situ. The cells were seeded in a glass-bottom cell sterile culture Petri dish specific for confocal microscopy (diameter of $15 \mathrm{~mm}$, Nest Biotechnology Co., Ltd., Shanghai, China) for 24 h. Following $24 \mathrm{~h}$ of treatment with glutamate or GLGZD, the cells were incubated with a mixture of $500 \mu 1 \mathrm{JC}-1$ staining fluid and $500 \mu \mathrm{l}$ cell culture medium in the dark at $37^{\circ} \mathrm{C}$ for $30 \mathrm{~min}$. Subsequently, the cells were washed twice with staining buffer preserved in $4^{\circ} \mathrm{C}$. Lastly, $1 \mathrm{ml}$ of cell culture medium was added to each specimen and the cells were analyzed using a LSM 710 laser scanning confocal microscope (Carl Zeiss, Oberkochen, Germany).

Analysis of $m R N A$ expression by reverse transcription polymerase chain reaction (RT-PCR). Total RNA was isolated using TRIzol reagent (Invitrogen Life Technologies, Carlsbad, CA, USA) according to the supplier's instructions. RNA was quantified by optical density measurements at 260 and $280 \mathrm{~nm}$. Integrity was confirmed by $1 \%$ agarose gel electrophoresis. We used $2 \mu \mathrm{g}$ of RNA in a $20 \mu 1$ reaction mixture utilizing M-MLV reverse transcriptase (Fermentas, Waltham, MA, USA) according to the supplier's instructions. The resultant reverse transcription products were stored at $-20^{\circ} \mathrm{C}$ until further use. Mouse polymer, $\mathrm{Bcl}-2$ and Bax primers were synthesized by Shanghai Sangon Biological Engineering Technology and Services Co.,Ltd. (Shanghai, China) according to the following sequences: Bax sense, 5'-GAGACACCTGA GCTGACCTTG-3' and antisense, 5'-GAAGTTGCCATCAG CAAACAT-3'; Bcl-2 sense, 5'-ATGTGTGTGGAGAGCGT CAAC-3' and antisense, 5'-CAGCCAGGAGAAATCAAA CAG-3'; $\beta$-actin sense, 5'-GAGACACCTGAGCTGACC TTG-3' and antisense, 5'-GAGACACCTGACCACCCTG TTGCTGTA-3'. The product size for Bax, Bcl-2 and $\beta$-actin sense was 195, 177 and $490 \mathrm{bp}$, respectively. PCR was carried out with Taq polymerase (Thermo Fisher Scientific Inc., Rockford, IL, USA) according to the supplier's instructions. The PCR conditions were as follows: $94^{\circ} \mathrm{C}$ for $4 \mathrm{~min}$, followed by 35 cycles ( 30 for $\beta$-actin) of 1 min denaturation at $94^{\circ} \mathrm{C}$, 1 min annealing at $58^{\circ} \mathrm{C}, 1$ min polymerization at $72^{\circ} \mathrm{C}$, and finally $10 \mathrm{~min}$ extension at $72^{\circ} \mathrm{C}$. The PCR products were 
A

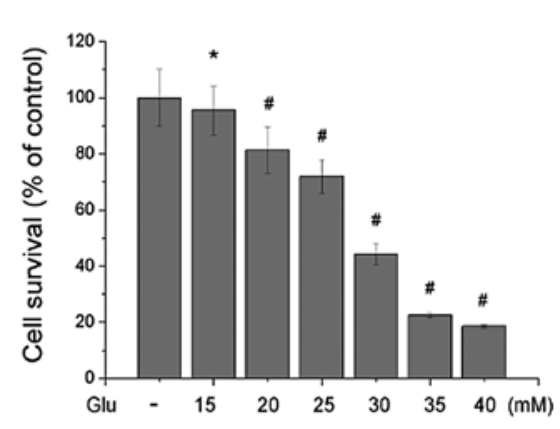

B

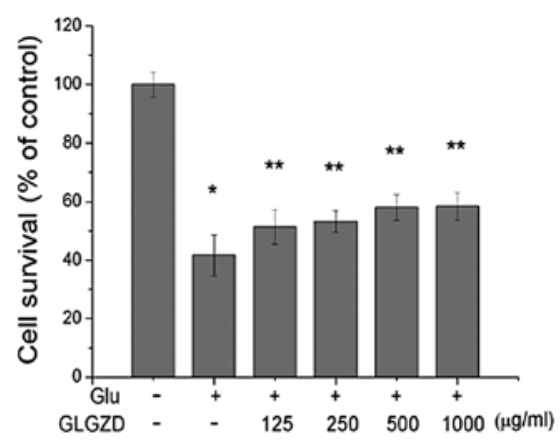

Figure 1. Effects of glutamate and Gua Lou Gui Zhi decoction (GLGZD) on BV-2 cell viability. (A) Cells were incubated for 24 h in media containing various concentrations of glutamate. Each value, calculated as a percentage of the 3-(4,5-dimethylthiazol-2-yl)-2,5-diphenyltetrazolium bromide (MTT) activity in the control (non-glutamate-treated) group, represents the mean $\pm \mathrm{SEM}(\mathrm{n}=12)$. $\mathrm{P}<0.05$ and ${ }^{\#} \mathrm{P}<0.01$ as compared with the control group. (B) Following treatment with $30 \mathrm{mM}$ glutamate for $24 \mathrm{~h}$, cells were treated with various concentrations of GLGZD for $24 \mathrm{~h} .{ }^{*} \mathrm{P}<0.01$ as compared with the control group; ${ }^{* *} \mathrm{P}<0.05$, as compared with the group treated only with glutamate. Glu, glutamate.

analyzed by $1.5 \%$ agarose electrophoresis and visualized using ethidium bromide $(0.25 \mu \mathrm{g} / \mathrm{ml})$ in $0.5 \mathrm{X}$ TBE buffer (Tris $40 \mathrm{mM}$, EDTA $1 \mathrm{mM}$, boric acid $44 \mathrm{mM}$ ) at $80 \mathrm{~V}$ (constant voltage). Images of gels were acquired and analyzed by Molecular Imager software (Bio-Rad Laboratories). The density of the PCR bands was expressed as a ratio of the band density divided by that of the housekeeping gene, $\beta$-actin.

Western blot analysis of protein expression. Cells were harvested and lysed in RIPA buffer containing the protease inhibitor, phenylmethylsulfonyl fluoride (PMSF) (both from Beyotime, Shanghai, China). Cell lysates were collected by centrifugation at $12,000 \mathrm{x}$ g for $10 \mathrm{~min}$ at $4^{\circ} \mathrm{C}$. Protein concentrations were determined using an enhanced BCA protein assay kit (Beyotime). Equal amounts of protein from each sample (40 $\mu \mathrm{g})$ were resolved by sodium dodecyl sulfate (SDS)-polyacrylamide gel electrophoresis (12\%) and transferred onto PVDF membranes. The membranes were incubated in blocking buffer (non-fat milk) and then incubated overnight at $4^{\circ} \mathrm{C}$ with rabbit polyclonal antibodies against Bax (CST, 1:1,000, $20 \mathrm{kDa}$ ), Bcl-2 (CST, 1:1,000, $26 \mathrm{kDa}$ ) or $\beta$-actin (1:4,000, $43 \mathrm{kDa})$ (Beyotime). The membranes were stringently washed and incubated with HRP-conjugated secondary antibodies (Proteintech Group, Chicago, IL, USA), for $1 \mathrm{~h}$ at room temperature. After washing, proteins were detected using enhanced chemiluminescence, and images were acquired using a Bio-Image Analysis System (Bio-Rad Laboratories).

Detection of cleaved caspase-3 protein by immunofluorescence. Cells were seeded in a glass-bottom cell sterile culture Petri dish specific for confocal microscopy (diameter of $15 \mathrm{~mm}$, Nest Biotechnology Co., Ltd.) for $24 \mathrm{~h}$. Following treatment with glutamate or GLGZD $(125,250,500$ and $1,000 \mu \mathrm{g} / \mathrm{ml}$ ) for $24 \mathrm{~h}$, the cells were fixed with $4 \%$ formaldehyde in PBS for $30 \mathrm{~min}$ at room temperature, rinsed three times in PBS, and incubated in blocking buffer $(5 \%$ BSA, $10 \%$ normal donkey serum, $0.3 \%$ Triton X-100 in PBS) for $60 \mathrm{~min}$ at room temperature. The cells were then incubated with cleaved caspase-3 antibody (Alexa Fluor 488-conjugated) overnight at $4^{\circ} \mathrm{C}(\mathrm{CST}, 1: 100)$. The cells were washed twice with PBS, and incubated with a rhodamine-conjugated phal- loidin (F-actin) antibody (cytoskeleton, $1: 100)$ at $37^{\circ} \mathrm{C}$ for $30 \mathrm{~min}$. Lastly, DAPI (Beyotime, 1:1,000) was added to each specimen for nuclei staining and the cells were analyzed using a LSM 710 laser scanning confocal microscope under a x40 water objective (Carl Zeiss).

Statistical analysis. Data are expressed as the means \pm standard error of mean (SEM). Data were first analyzed using Portable IBM SPSS Statistics software. A paired-sample t-test was then performed to compare the treated samples, and values of $\mathrm{P}<0.05$ were considered to indicate statistically significant differences.

\section{Results}

Total phenolic compound and sugar content in the extract. In our previous study (6), a high-performance liquid chromatography (HPLC) fingerprint was used to control the quality of the GLGZD extract, which revealed that the method we use to prepare GLGZD was efficient and that the product used for this study was pure.

Effect of GLGZD on viability and apoptosis in glutamatestimulated $B V-2$ cells. In order to evaluate the effects of GLGZD on glutamate-induced cell death, we first determined the optimal concentration of glutamate which was able to induce BV-2 cell death (Fig. 1A). Increasing concentrations of glutamate were added $(15,20,25,30,35$ and $40 \mathrm{mM})$ and cell survival was measured. The concentration of glutamate that induced approximately $50 \%$ cell death, and the appropriate concentration for our experiments, was $30.0 \mathrm{mM}$.

The glutamate-induced loss of cell viability was markedly attenuated by treatment with GLGZD (Fig. 1B). Following treatment with $30 \mathrm{mM}$ glutamate for $24 \mathrm{~h}$, cells survived for an average of $41.72 \pm 6.95 \%$ of the control value. Treatment with $125,250,500$ and $1,000 \mu \mathrm{g} / \mathrm{ml}$ of GLGZD in the presence of $30.0 \mathrm{mM}$ glutamate markedly increased cell viability to $51.37 \pm 5.99,53.24 \pm 3.68,58.02 \pm 4.34$ and $58.39 \pm 4.81 \%$, respectively. These results demonstrated that the glutamate-induced loss of cell viability was partially attenuated by GLGZD in a dose-dependent manner. 

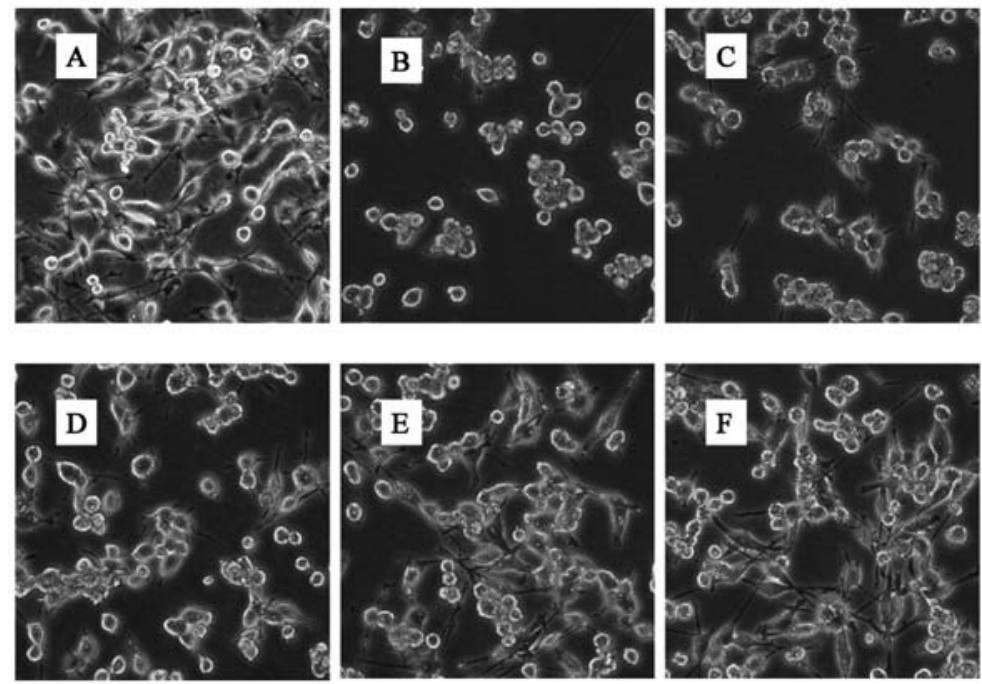

Figure 2. Effect of glutamate (Glu) treatment and Gua Lou Gui Zhi decoction (GLGZD) on cell morphology of BV-2 cells. (A) Control, (B) Glu, (C) Glu + GLGZD (125 $\mu \mathrm{g} / \mathrm{ml})$, (D) Glu + GLGZD (250 $\mu \mathrm{g} / \mathrm{ml})$, (E) Glu + GLGZD (500 $\mu \mathrm{g} / \mathrm{ml})$, (F) Glu + GLGZD (1,000 $\mu \mathrm{g} / \mathrm{ml}) ;$ (magnification, x20).

A

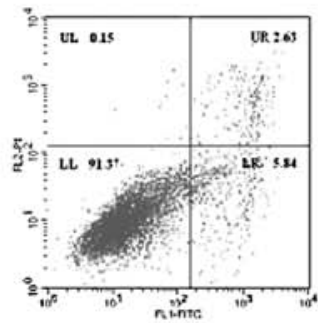

D

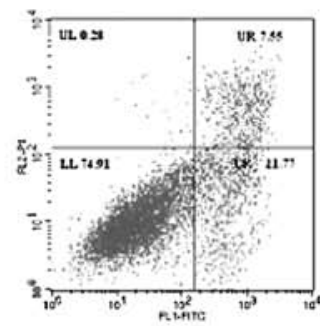

B

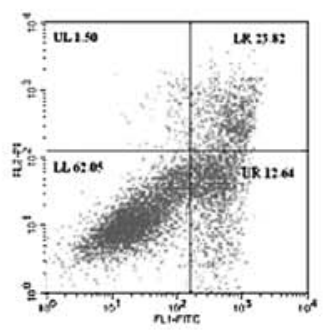

$\mathrm{E}$

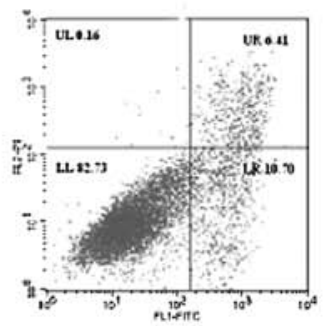

C

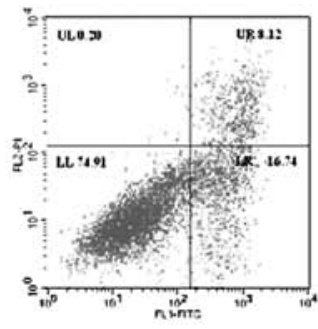

$\mathrm{F}$

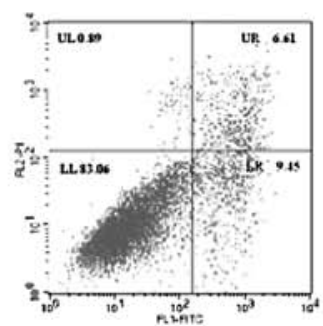

G

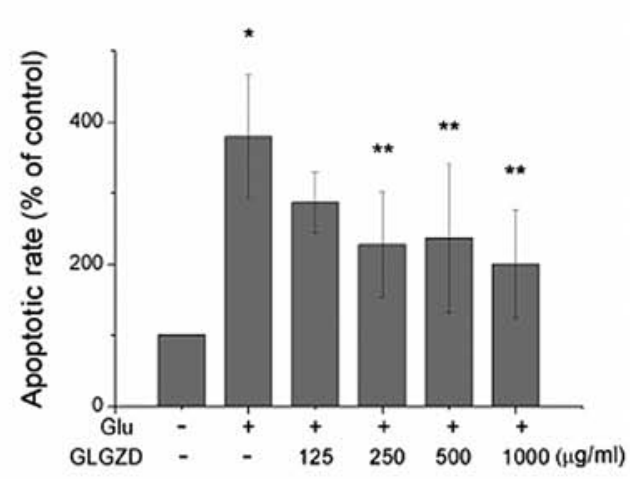

Figure 3. Effect of Gua Lou Gui Zhi decoction (GLGZD) on cell apoptosis of BV-2 cells. Cells were collected, stained with Annexin V/propidium iodide (PI) and analyzed by flow cytometry. (A) Control, (B) glutamate (Glu), (C) Glu + GLGZD (125 $\mu \mathrm{g} / \mathrm{ml})$, (D) Glu + GLGZD (250 $\mu \mathrm{g} / \mathrm{ml})$, (E) Glu + GLGZD (500 $\mu \mathrm{g} / \mathrm{ml})$, (F) Glu + GLGZD (1,000 $\mu \mathrm{g} / \mathrm{ml}$ ). Upper right quadrant, Annexin V/PI double-positive stained cells (late apoptosis); lower right quadrant, Annexin V-positive/ PI-negative stained cells (early apoptosis). (G) Results from flow cytometric analysis are expressed as the means \pm SEM of three independent experiments. The apoptotic rate was calculated using Annexin V/PI double-positive stained cell plus Annexin V-positive/PI-negative stained cell populations. ${ }^{*} \mathrm{P}<0.01$ as compared with control group. ${ }^{* * *} \mathrm{P}<0.05$, compared with the group treated only with glutamate.

In addition to cell viability, we also sought to analyze the morphological characteristics of BV-2 cells cultured in the presence of glutamate with or without GLGZD. BV-2 cells display a characteristic small spherical morphology with more than half of the cells displaying process-bearing sites, often bipolar and tripolar (Fig. 2A). The addition of $30 \mathrm{mM}$ glutamate induced contraction, rounding and even floating of the majority of cells (Fig. 2B). This suggests the involvement of microglial cell apoptosis and necrosis induced by treatment with glutamate. This morphological change was effectively inhibited by 250,500 and $1,000 \mu \mathrm{g} / \mathrm{ml}$ of GLGZD (Fig. 2D-F).
A quantitative evaluation of apoptosis was then carried out by flow cytometry with an Annexin V/PI test. The apoptotic rate of the cells treated with $30 \mathrm{mM}$ glutamate alone for $24 \mathrm{~h}$ markedly increased to $36.46 \%$ (Fig. 3). However, treatment with GLGZD reversed this effect. The proportion of apoptotic cells decreased from $36.46 \%$ to $24.86,19.32,17.11$ and $16.06 \%$, when the cells were co-incubated with concentrations of 125 , 250,500 and $1,000 \mu \mathrm{g} / \mathrm{ml}$ of GLGZD, respectively (Fig. 3A-F). A dose-dependent effect was evident, as the highest concentration of GLGZD $(1,000 \mu \mathrm{g} / \mathrm{ml})$ demonstrated the least number of apoptotic cells (Fig. 3G). 
A

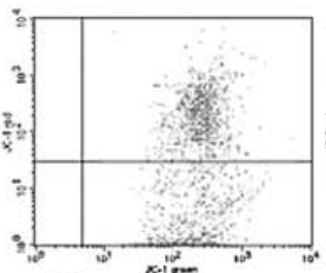

D

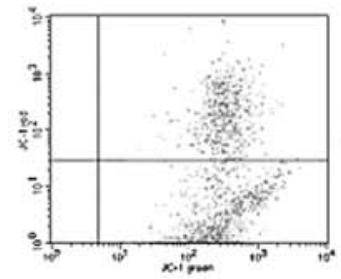

B

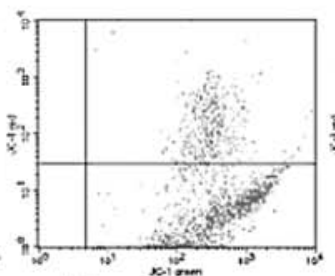

$\mathrm{E}$

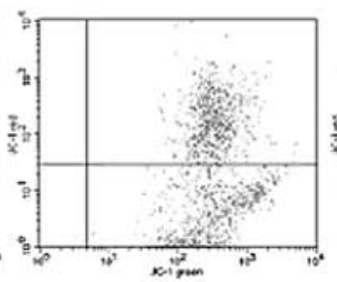

C

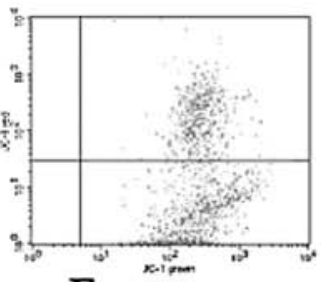

$\mathrm{F}$

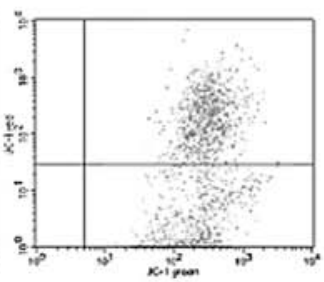

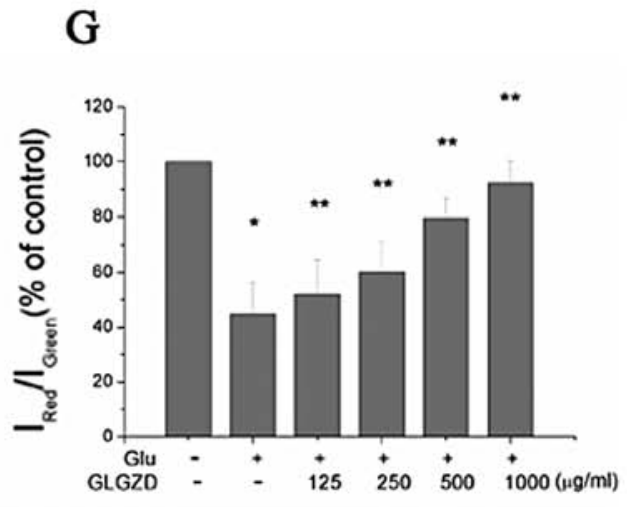

Figure 4. Effect of Gua Lou Gui Zhi decoction (GLGZD) on mitochondrial membrane potential (MMP) of BV-2 cells by flow cytometry. A representative dot plot of the changed MMP by flow cytometry after the labeling of fluorescent probe with JC-1 in both FL-1 and FL-2 channels. (A) Control, (B) glutamate (Glu), (C) Glu + GLGZD (125 $\mu \mathrm{g} / \mathrm{ml})$, (D) Glu + GLGZD (250 $\mu \mathrm{g} / \mathrm{ml})$, (E) Glu + GLGZD (500 $\mu \mathrm{g} / \mathrm{ml})$, (F) Glu + GLGZD (1,000 $\mu \mathrm{g} / \mathrm{ml})$. (G) The quantitative MMP from each group is expressed as the ratio of red fluorescence intensity over the green fluorescence intensity. The data are expressed as the means \pm SEM from three independent experiments. ${ }^{*} \mathrm{P}<0.01$ as compared with the control group; ${ }^{* *} \mathrm{P}<0.05$, compared with the group treated only with glutamate.
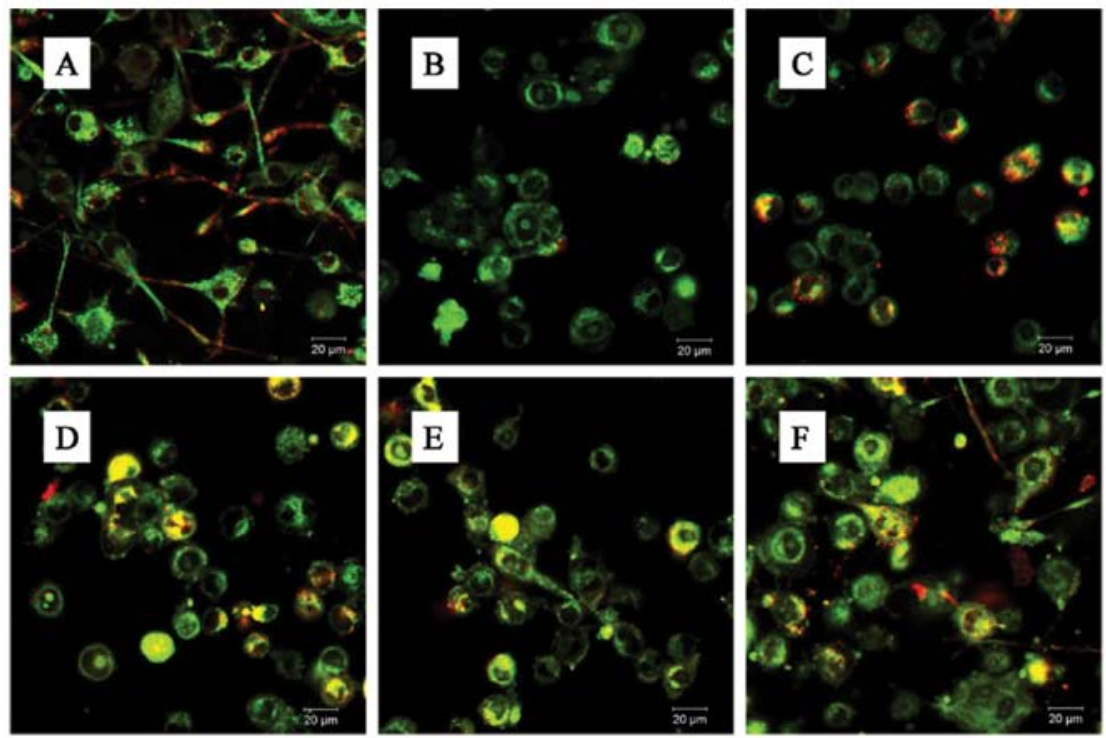

Figure 5. Effect of Gua Lou Gui Zhi decoction (GLGZD) on mitochondrial membrane potential (MMP) of BV-2 cells, as shown by fluorescence. A fluorescence photomicrograph of in situ JC-1 staining output by laser scanning confocal microscopy (magnification, $\mathrm{x} 40$; scale bar, 20 $\mu \mathrm{m}$ ). (A) Control, (B) glutamate (Glu), (C) Glu + GLGZD (125 $\mu \mathrm{g} / \mathrm{ml}),(\mathrm{D}) \mathrm{Glu}+\mathrm{GLGZD}(250 \mu \mathrm{g} / \mathrm{ml}),(\mathrm{E}) \mathrm{Glu}+\mathrm{GLGZD}(500 \mu \mathrm{g} / \mathrm{ml}),(\mathrm{F}) \mathrm{Glu}+\mathrm{GLGZD}(1,000 \mu \mathrm{g} / \mathrm{ml})$.

Effects of GLGZD on MMP in glutamate-stimulated BV-2 cells. Apoptosis is often accompanied by mitochondrial dysfunction, and the decline in MMP is considered as a symbolic event of early cellular apoptosis $(14,15)$. In this study, to investigate the effects of glutamate and GLGZD on mitochondrial function, indicators of mitochondrial activity were monitored using JC-1 staining. The MMP of BV-2 cells treated with glutamate for $24 \mathrm{~h}$ was markedly reduced (Fig. 4). The ratio of aggregated JC-1 (FL-2 channel) to monomeric JC-1 (FL-1 channel) was decreased from 79.8 (control group) to $33.7 \%$ (glutamatetreated only group) $(\mathrm{P}<0.01)$ after $24 \mathrm{~h}$ of treatment. When the cells were incubated with $125,250,500$ and $1,000 \mu \mathrm{g} / \mathrm{ml}$ of GLGZD, the ratio increased from $33.7 \%$ to $42.7,54.5,78.4$ and $79.7 \%$, respectively (Fig. 4A-F). Treatment with GLGZD attenuated the decline in MMP in a dose-dependent manner, as indicated by the increase in red (JC-1 aggregates)/green (JC-1 monomers) ratio (Fig. 4G).

In addition, the BV-2 cells stained with JC-1 exhibited mitochondrial red fluorescence with a little green fluorescence, suggesting that the cells were in a normal polarized state (Fig. 5A). The JC-1 aggregates were dispersed to the monomeric form (green fluorescence) in the glutamate-treated cells (Fig. 5B). However, treatment with GLGZD attenuated the dissipation of the MMP (Fig. 5C-F), corroborating our results from flow cytometry (Fig. 4).

Effects of GLGZD on the mRNA and protein expression levels of Bax and Bcl-2 in glutamate-stimulated BV-2 cells. 

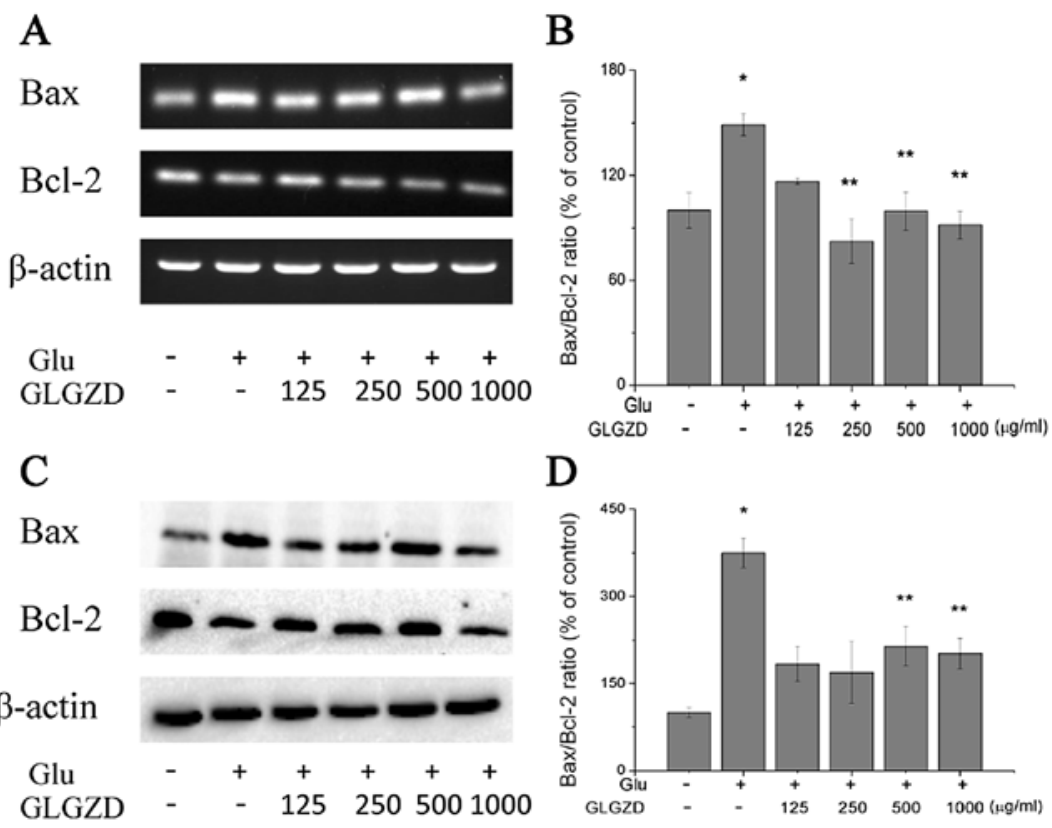

Figure 6. Effects of Gua Lou Gui Zhi decoction (GLGZD) on the mRNA and protein expression levels of Bax/Bcl-2 in BV-2 cells. (A) The mRNA expression levels of Bax and Bcl-2 were quantified by densitometric analysis and (B) the Bax/Bcl-2 ratio was determined. (C) The protein expression levels of Bax and Bcl-2 were also quantified by densitometric analysis and (D) the Bax/Bcl-2 ratio was determined at the protein level. Data are the means \pm SEM of three independent experiments in triplicate. ${ }^{*} \mathrm{P}<0.01$ as compared with the control group; ${ }^{* *} \mathrm{P}<0.05$, compared the group treated only with glutamate (Glu).

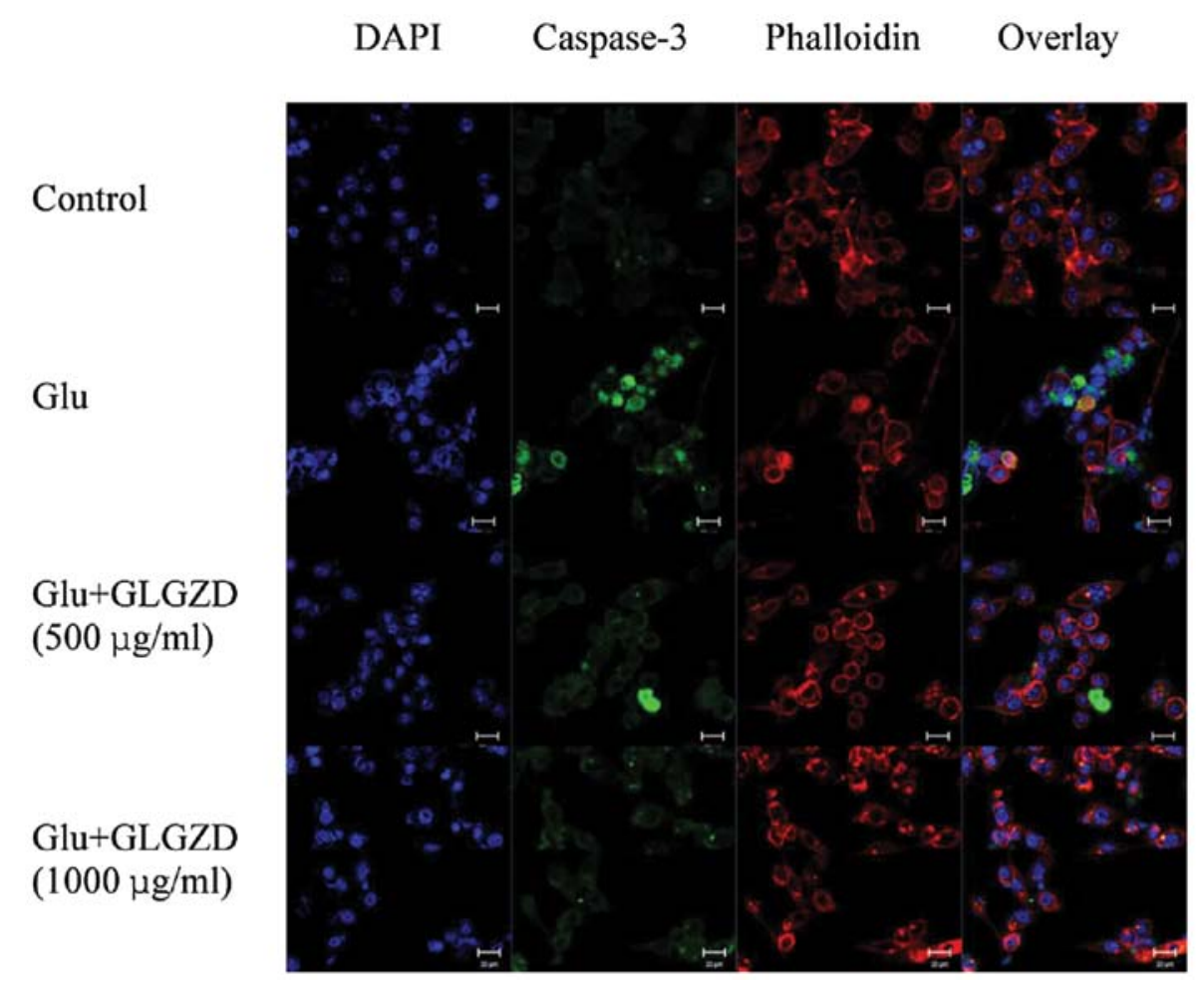

Figure 7. Effects of Gua Lou Gui Zhi decoction (GLGZD) on the expression of caspase-3 protein in BV-2 cells. Caspase-3 was labeled with cleaved caspase-3 antibody (Alexa Fluor 488-conjugated, green). Nuclear counterstaining signals with DAPI (blue) and F-actin cytoskeleton labeled with rhodamine phalloidin (red) are shown (magnification, $\mathrm{x} 40$; scale bar, $20 \mu \mathrm{m}$ ). Glu, glutamate.

To determine whether GLGZD protects the BV-2 cells from glutamate-induced apoptosis by modulating the Bcl-2 family of proteins, the mRNA and protein levels of Bax and Bcl-2 were estimated using RT-PCR and western blot analysis. We detected an increase in Bax and a decrease in Bcl-2 mRNA levels following exposure to glutamate (Fig. 6A and B). 
Treatment with GLGZD inhibited the upregulation of Bax and enhanced the upregulation of Bcl-2 slightly at $24 \mathrm{~h}$ of glutamate exposure. Thus, GLGZD attenuated the increase in the Bax to $\mathrm{Bcl}-2$ ratio induced by glutamate, a sign of apoptosis inhibition. In addition to the GLGZD modulation of Bax and Bcl-2 mRNA levels, we observed similar results with the protein levels (Fig. 6C and D).

Effects of GLGZD on the expression of cleaved caspase-3 protein in glutamate-stimulated $B V-2$ cells. We utilized an antibody specific for the activated form of caspase-3, a caspase that plays an important role in a number of neuronal apoptotic pathways, as an 'executioner' of cell death (16). Treatment with glutamate led to the formation of apoptotic nuclei, as assessed by an abundance of green puncta labeled with the antibody to activated caspase-3 (Fig. 7). Cleaved caspase-3 was localized in the nuclei, as it overlapped with apoptotic cell nuclei. Treatment with GLGZD induced the re-localization of caspase-3, out of the nucleus, representing a decrease in caspase-3 expression levels (Fig. 7)

\section{Discussion}

Increasing evidence indicates that a number of herbal medicinal plants, including some formulations used in TCM, have beneficial effects on neurodegenerative diseases, such as Artemisia annua L. (17), baicalein $(2,14)$, cassia twig (18), 6-Shogaol (a ginger product) (19), Gastrodia elata Blume. (20), Chrysanthemum indicum Linné (21), pinocembrin (11), Buyang Huanwu decoction (22), Guizhi-Fuling capsules (23), Xiao-Xu-Ming decoction (24), Danggui-Shaoyao-San (25) and Yi-Gan San (13). Similar to other Chinese medicinal compounds, GLGZD is thought to possess various traditional and ethnopharmacological benefits for neurodegenerative diseases, and it has long been clinically employed in the treatment of stroke (5). Although the underlying mechanisms remain largely unknown, a rat model of focal cerebral ischemia-reperfusion (I/R) injury demonstrated that GLGZD exerts neuroprotective and anti-spasticity effects in a model of cerebral ischemia through the modulation of glutamate levels and $\alpha$-amino-3-hydroxy-5-methyl-4-isoxazolepropionic acid (AMPA) receptor expression (6). In addition, GLGZD has been shown to induce an anti-inflammatory response through the suppression of the LPS-stimulated TLR4/NF- $\kappa$ B pathway in BV-2 murine microglial cells (7). However, molecular studies on cellular models of toxin-based Parkinson's disease have suggested that oxidative stress-mediated mitochondrial dysfunction, apoptosis and microglial-mediated neuroinflammation play a major etiological role in neurotoxicity (26). Therefore, in the present study, we focused on the ability of GLGZD to suppress neurodegeneration and neuroinflammation in a cellular model of glutamate-induced apoptosis and to explore the intrinsic mechanisms involved.

We examined the neuroprotective effects of GLGZD on the glutamate-induced apoptosis of BV-2 cells. Glutamate significantly decreased cell viability and increased cell apoptosis. An MTT assay and an Annexin V/PI test revealed that GLGZD markedly inhibited the glutamate-induced apoptosis of BV-2 cells. The Annexin V/PI test results revealed that glutamate increased early and late apoptosis; however, treatment with GLGZD suppressed the percentage of early apoptotic cells.

Genes of the Bcl-2 family play a key role in the mitochondrial pathway of apoptosis, reflecting the balance between the pro- and anti-apoptotic members of the Bcl-2 family, of which Bax and Bcl-2 are the two main members (27). In this study, we found that glutamate had a profound effect on the gene expression and protein levels of Bax and Bcl-2. Our results indicated that GLGZD provided neuroprotection partly by inhibiting Bax overexpression and increasing anti-apoptotic Bcl-2 expression. Treatment with GLGZD reduced the expression of pro-apoptotic Bax and increased the expression of anti-apoptotic Bcl-2 significantly in a dose-dependent manner, thereby attenuating the elevated glutamate-induced $\mathrm{Bax} / \mathrm{Bcl}-2$ ratio in the BV-2 cells. This finding indicate that the protective effects of GLGZD against neurological damage are likely attributed to its anti-apoptotic properties.

Caspases are cysteine proteases that are essential for apoptosis in a variety of in vitro and in vivo models. Caspase- 3 is the major executioner protease, responsible for initiating the mitochondrial-regulated apoptotic program $(16,27,28)$. We found that the levels of caspase-3, which is normally expressed at low levels, increased significantly when the cells were treated with glutamate for $24 \mathrm{~h}$. However, following treatment with GLGZD, the increased expression levels of caspase-3 were slightly lower, and these changes occurred in a dosedependent manner.

The activation of microglial cells plays a crucial role in the initiation and progression of brain inflammation, and BV-2 cells have been used to study the expression of various proinflammatory and anti-inflammatory cytokines (8). Although in a previous study, we revealed that GLGZD exhibited an antiinflammatory response on LPS-induced BV-2 cell damage (7), it remains unknown whether the pro-apoptotic effects of glutamate are due to the excitotoxic properties of pro-inflammatory cytokines or to the direct activation of microglial phagocytosis (4). Therefore, the similarity and differences between the possible anti-apoptotic and anti-inflammatory mechanisms of GLGZD on microglial cells require further investigation.

In conclusion, GLGZD exerts protective effects against glutamate-induced cellular injury. To our knowledge, this is the first report revealing the role of GLGZD in protecting BV-2 cells against glutamate-induced neurotoxicity. Further studies on mature primary neurons, as well as on animal models of Parkinson's disease and comparisons with known anti-parkinsonian agents are, however, required to establish both efficacy and safety. Based on the protective effects of GLGZD on glutamate-induced cellular injury, GLGZD may be used as a potential therapeutic candidate for the treatment of neurodegenerative disorders.

\section{Acknowledgements}

This study was sponsored by the National Natural Science Foundation of China (no. 81273835), the Guidance Project of the Fujian Provincial Department of Science and Technology (no. 2012D012), the Key Project of Fujian Provincial Department of Science and Technology (no. 2012Y0041), the Project of Fujian Education Department (no. JK2012024), and the Project of Fujian Education Department (no. JA2012179). 


\section{References}

1. Eun SY, Hong YH, Kim EH, Jeon H, Suh YH, Lee JE, Jo C, Jo SA and Kim J: Glutamate receptor-mediated regulation of c-fos expression in cultured microglia. Biochem Biophys Res Commun 325: 320-327, 2004.

2. Hwang KY, Oh YT, Yoon H, Lee J, Kim H, Choe W and Kang I: Baicalein suppresses hypoxia-induced HIF-1alpha protein accumulation and activation through inhibition of reactive oxygen species and PI 3-kinase/Akt pathway in BV2 murine microglial cells. Neurosci Lett 444: 264-269, 2008.

3. Kim BW, Koppula S, Kim JW, Lim HW, Hwang JW, Kim IS, Park PJ and Choi DK: Modulation of LPS-stimulated neuroinflammation in BV-2 microglia by Gastrodia elata: 4-hydroxybenzyl alcohol is the bioactive candidate. J Ethnopharmacol 139: 549-557, 2012.

4. Tremblay MÈ, Stevens B, Sierra A, Wake H, Bessis A and Nimmerjahn A: The role of microglia in the healthy brain. J Neurosci 31:16064-16069, 2011.

5. Chen YL, Chen LD and Tao J: Clinical research on treating limbs spasm from cerebral apoplexy with the Gualou Guizhi decoction. Clin J Chin Med 5: 7-9, 2013 (In Chinese).

6. Huang J, Tao J, Xue X, Yang S, Han P, Lin Z, Xu W, Lin J, Peng J and Chen L: Gua Lou Gui Zhi decoction exerts neuroprotective effects on post-stroke spasticity via the modulation of glutamate levels and AMPA receptor expression. Int J Mol Med 31: 841-848, 2013.

7. Hu H, Li Z, Zhu X, Lin R, Lin J, Peng J, Tao J and Chen L: Gua Lou Gui Zhi decoction suppresses LPS-induced activation of the TLR4/NF-кB pathway in BV-2 murine microglial cells. Int J Mol Med 31: 1327-1332, 2013.

8. Lin HC, Yang CM, Liu CL and Hu ML: Synergistic effects of homocysteine, S-adenosylhomocysteine and adenosine on apoptosis in BV-2 murine microglial cells. BioFactors 34: 81-95, 2008.

9. Lin CH, Kuo SC, Huang LJ and Gean PW: Neuroprotective effect of $\mathrm{N}$-acetylcysteine on neuronal apoptosis induced by a synthetic gingerdione compound: involvement of ERK and p38 phosphorylation. J Neurosci Res 84: 1485-1494, 2006.

10. Platt SR: The role of glutamate in central nervous system health and disease-a review. Vet J 173: 278-286, 2007.

11. Gao M, Zhang WC, Liu QS, Hu JJ, Liu GT and Du GH: Pinocembrin prevents glutamate-induced apoptosis in SH-SY5Y neuronal cells via decrease of bax/bcl-2 ratio. Eur J Pharmacol 591: 73-79, 2008.

12. Hu Y, Li J, Liu P, Chen X, Guo DH, Li QS and Rahman K: Protection of SH-SY5Y neuronal cells from glutamate-induced apoptosis by 3,6'-disinapoyl sucrose, a bioactive compound isolated from Radix Polygala. J Biomed Biotechnol: 1-5, 2012.

13. Kawakami Z, Kanno H, Ikarashi Y and Kase Y: Yokukansan, a kampo medicine, protects against glutamate cytotoxicity due to oxidative stress in PC12 cells. J Ethnopharmacol 134: 74-81, 2011.

14. Zhang SH, Ye JL and Dong GX: Neuroprotective effect of baicalein on hydrogen peroxide- mediated oxidative stress and mitochondrial dysfunction in PC12 cells. J Mol Neurosci 40: 311-320, 2010.
15. Zhang Y, Ma H, Xie B, Han C, Wang C, Qing H and Deng Y: Alpha-synuclein overexpression induced mitochondrial damage by the generation of endogenous neurotoxins in PC12 cells. Neurosci Lett 547: 65-69, 2013

16. Sharifi AM, Eslami H, Larijani B and Davoodi J: Involvement of caspase-8, -9 , and -3 in high glucose-induced apoptosis in PC12 cells. Neurosci Lett 459: 47-51, 2009.

17. Lee IS, Ryu DK, Lim J, Cho S, Kang BY and Choi HJ: Artesunate activates $\mathrm{Nrf} 2$ pathway-driven anti-inflammatory potential through ERK signaling in microglial BV2 cells. Neurosci Lett 509: 17-21, 2012.

18. Sui F, Lin N, Guo JY, Zhang CB, Du XL, Zhao BS, Liu HB, Yang N, Li LF, Guo SY, Huo HR and Jiang TL: Cinnamaldehyde up-regulates the mRNA expression level of TRPV1 receptor potential ion channel protein and its function in primary rat DRG neurons in vitro. J Asian Nat Prod Res 12: 76-87, 2010.

19. Ha SK, Moon E, Ju MS, Kim DH, Ryu JH, Oh MS and Kim SY: 6-Shogaol, a ginger product, modulates neuroinflammation: a new approach to neuroprotection. Neuropharmacology 63: 211-223, 2012.

20. An H, Kim IS, Koppula S, Kim BW, Park PJ, Lim BO, Choi WS, Lee KH and Choi DK: Protective effects of Gastrodia elata Blume on $\mathrm{MPP}^{+}$-induced cytotoxicity in human dopaminergic SH-SY5Y cells. J Ethnopharmacol 130: 290-298, 2010.

21. Kim IS, Ko HM, Koppula S, Kim BW and Choi DK: Protective effect of Chrysanthemum indicum Linne against 1-methyl4-phenylpridinium ion and lipopolysaccharide-induced cytotoxicity in cellular model of Parkinson's disease. Food Chem Toxicol 49: 963-973, 2011.

22. Zhao LD, Wang JH, Jin GR, Zhao Y and Zhang HJ: Neuroprotective effect of Buyang Huanwu decoction against focal cerebral ischemia/reperfusion injury in rats - time window and mechanism. J Ethnopharmacol 140: 339-344, 2012.

23. Li TJ, Qiu Y, Mao JQ, Yang PY, Rui YC and Chen WS: Protective effects of Guizhi-Fuling-Capsules on rat brain ischemia/reperfusion injury. J Pharmacol Sci 105: 34-40, 2007.

24. Zhu XH, Li SJ, Hu HH, Sun LR, Das M and Gao TM: Neuroprotective effects of Xiao-Xu-Ming decoction against ischemic neuronal injury in vivo and in vitro. $J$ Ethnopharmacol 127: 38-46, 2010.

25. Qian YF, Wang H, Yao WB and Gao XD: Aqueous extract of the Chinese medicine, Danggui-Shaoyao-San, inhibits apoptosis in hydrogen peroxide-induced PC12 cells by preventing cytochrome $\mathrm{c}$ release and inactivating of caspase cascade. Cell Biol Int 32: 304-311, 2008.

26. Block ML, Zecca L and Hong JS: Microglia-mediated neurotoxicity: uncovering the molecular mechanisms. Nat Rev Neurosci 8: 57-69, 2007.

27. Lang-Rollin I, Maniati M, Jabado O, Vekrellis K, Papantonis S, Rideout HJ and Stefanis L: Apoptosis and the conformational change of Bax induced by proteasomal inhibition of PC12 cells are inhibited by bcl-xL and bcl-2. Apoptosis 10: 809-820, 2005.

28. Ding F, Shao ZW, Yang SH, Wu Q, Gao F and Xiong LM: Role of mitochondrial pathway in compression-induced apoptosis of nucleus pulposus cells. Apoptosis 17: 579-590, 2012. 